Gläser, Eva; Krumbacher, Christina

\title{
Ausstattung zur technischen Bildung mangelhaft? Eine quantitative Studie zur Situation an Grundschulen
}

Landwehr, Brunhild [Hrsg.]; Mammes, Ingelore [Hrsg.]; Murmann, Lydia [Hrsg.]: Technische Bildung im Sachunterricht der Grundschule. Elementar bildungsbedeutsam und dennoch vernachlässigt? Bad Heilbrunn : Verlag Julius Klinkhardt 2021, S. 151-165. - (Forschungen zur Didaktik des Sachunterrichts; 12)

\section{Quellenangabe/ Reference:}

Gläser, Eva; Krumbacher, Christina: Ausstattung zur technischen Bildung mangelhaft? Eine quantitative Studie zur Situation an Grundschulen - In: Landwehr, Brunhild [Hrsg.]; Mammes, Ingelore [Hrsg.]; Murmann, Lydia [Hrsg.]: Technische Bildung im Sachunterricht der Grundschule. Elementar bildungsbedeutsam und dennoch vernachlässigt? Bad Heilbrunn : Verlag Julius Klinkhardt 2021, S. 151-165 - URN: urn:nbn:de:0111-pedocs-215377 - DOI: 10.25656/01:21537

https://nbn-resolving.org/urn:nbn:de:0111-pedocs-215377

https://doi.org/10.25656/01:21537

in Kooperation mit / in cooperation with:

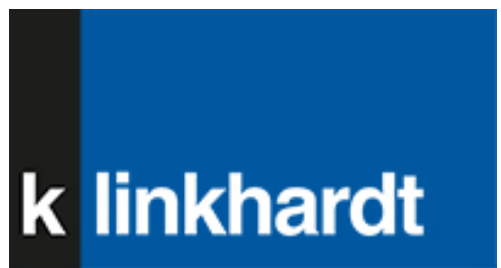

http://www.klinkhardt.de

\section{Nutzungsbedingungen}

Dieses Dokument steht unter folgender Creative Commons-Lizenz: http://creativecommons.org/licenses/by-nc-sa/4.0/deed.de - Sie dürfen das Werk bzw. den Inhalt unter folgenden Bedingungen vervielfältigen, verbreiten und öffentlich zugänglich machen sowie Abwandlungen und Bearbeitungen des Werkes bzw. Inhaltes anfertigen: Sie müssen den Namen des Autors/Rechteinhabers in der von ihm festgelegten Weise nennen. Dieses Werk bzw. der Inhalt darf nicht für kommerzielle Zwecke verwendet werden. Die neu entstandenen Werke bzw. Inhalte dürfen nur unter Verwendung von Lizenzbedingungen weitergegeben werden, die mit denen dieses Lizenzvertrages identisch oder vergleichbar sind.

Mit der Verwendung dieses Dokuments erkennen Sie die Nutzungsbedingungen an.

\section{Terms of use}

This document is published under following Creative Commons-License: http://creativecommons.org/licenses/by-nc-sa/4.0/deed.en - You may copy, distribute and transmit, adapt or exhibit the work in the public and alter, transform or change this work as long as you attribute the work in the manner specified by the author or licensor. You are not allowed to make commercial use of the work. If you alter, transform, or change this work in any way, you may distribute the resulting work only under this or a comparable license.

By using this particular document, you accept the above-stated conditions of use.

\section{Kontakt / Contact:}

\section{peDOCS}

DIPF | Leibniz-Institut für Bildungsforschung und Bildungsinformation Informationszentrum (IZ) Bildung

E-Mail: pedocs@dipf.de

Internet: www.pedocs.de

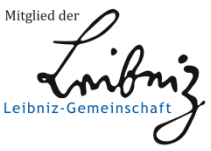


Forschungen zur Didaktik des Sachunterrichts

Brunhild Landwehr Ingelore Mammes

Lydia Murmann

(Hrsg.)

Technische Bildung

im Sachunterricht der Grundschule

Elementar bildungsbedeutsam und dennoch vernachlässigt? 
Forschungen zur Didaktik des Sachunterrichts

Band 12 


\section{Brunhild Landwehr Ingelore Mammes Lydia Murmann \\ (Hrsg.)}

\section{Technische Bildung im Sachunterricht der Grundschule}

Elementar bildungsbedeutsam und dennoch vernachlässigt? 
Schriftenreihe der

Gesellschaft für Didaktik des Sachunterrichts e.V.

Die Gesellschaft für Didaktik des Sachunterrichts (GDSU) e.V. ist ein Zusammenschluss von Lehrenden aus Hochschule, Lehrerfortbildung, Lehrerweiterbildung und Schule. Ihre Aufgabe ist die Förderung der Didaktik des Sachunterrichts als wissenschaftliche Disziplin in Forschung und Lehre sowie die Vertretung der Belange des Schulfaches Sachunterricht. www.gdsu.de

Dieser Titel wurde in das Programm des Verlages mittels eines Peer-Review-Verfahrens aufgenommen. Für weitere Informationen siehe www.klinkhardt.de.

Bibliografische Information der Deutschen Nationalbibliothek Die Deutsche Nationalbibliothek verzeichnet diese Publikation in der Deutschen Nationalbibliografie; detaillierte bibliografische Daten sind im Internet abrufbar über http://dnb.d-nb.de.

2021.n. () by Julius Klinkhardt.

Druck und Bindung: AZ Druck und Datentechnik, Kempten.

Printed in Germany 2021.

Gedruckt auf chlorfrei gebleichtem alterungsbeständigem Papier.

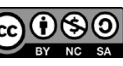

Die Publikation (mit Ausnahme aller Fotos, Grafiken und Abbildungen) ist veröffent-

licht unter der Creative Commons-Lizenz: CC BY-NC-SA 4.0 International

https://creativecommons.org/licenses/by-nc-sa/4.0/

ISBN 978-3-7815-5869-4 digital

doi.org/10.35468/5869

ISBN 978-3-7815-2430-9 print 


\section{Inhaltsverzeichnis}

Brunhild Landwehr, Ingelore Mammes und Lydia Murmann

Editorial

Andreas Schmitt und Tanja Fellensiek

„Windräder werden mit Strom betrieben, um Wind zu erzeugen ...

oder umgekehrt!?" - Schülervorstellungen und Konzeptwechsel zum

Thema Windenergie im Sachunterricht

Stefan Fletcher und Anja Kleinteich

Vorstellungen von Grundschüler*innen zum Ende der Primarstufe über den grundsätzlichen Aufbau eines komplexen technischen Systems zur Energieerzeugung untersucht am Beispiel der Konstruktion eines Wasserkraftwerks aus vorgegebenen Teilsystemen

Swantje Dölle

LERNnetze - Lernunterstützung im technischen Sachunterricht

Erprobung kognitiv aktivierender und inhaltlich strukturierender

Maßnahmen der Lernunterstützung und

Überprüfung der Angebotsnutzung

\section{Victoria Adenstedt}

Attributionen von Grundschulkindern zur Erklärung von

Leistungsergebnissen bei technischen Alltagsaufgaben

\section{Svantje Schumann}

Technische Ereignisse in Stummfilmen erschließen -

eine Untersuchung der Bildungsprozesse von Kindern

Lennart Goecke, Jurik Stiller und Julia Schwanewedel

Algorithmusverständnis in der Primarstufe - Eine Studie im Kontext

des Einsatzes von programmierbarem Material

Sabine Martschinke, Susanne Palmer Parreira und Ralf Romeike

Informatische (Grund-)Bildung schon in der Primarstufe?

Erste Ergebnisse aus einer Evaluationsstudie 
6| Inhaltsverzeichnis

Eva Gläser und Christina Krumbacher

Ausstattung zur technischen Bildung mangelhaft?

Eine quantitative Studie zur Situation an Grundschulen

Verzeichnis der Autorinnen und Autoren ............................................... 167 
Eva Gläser und Christina Krumbacher

\section{Ausstattung zur technischen Bildung mangelhaft? Eine quantitative Studie zur Situation an Grundschulen}

\section{Einleitung}

Die Relevanz technischer Bildungsprozesse in der Grundschule wurde in den letzten Jahren vermehrt erläutert und begründet (vgl. z. B. Mammes \& Tuncsoy, 2013, Möller, 2014, Möller, 2015). Ein Schwerpunkt, der hierbei vor allem diskutiert wurde, ist die konzeptionelle Ausrichtung der Technischen Bildung. Auch im „Perspektivrahmen Sachunterricht“ der Fachgesellschaft GDSU (2013) wird insbesondere das „Bildungspotenzial“ der „technischen Perspektive: Technik-Arbeit“ (63ff.) näher ausgeführt. Grundlegend ist, dass im Sachunterricht sowohl perspektivenbezogene Themen als auch perspektivenbezogene Denk, Arbeits- und Handlungsweisen der technischen Perspektive integrieren werden. „Mit den Prozessen der Problemfindung, des praktischen Handelns, Erkundens, Konstruierens, Optimierens und Bewertens" soll Schüler*innen ermöglicht werden, sich Technik zu erschließen. Betont wird zudem: „Technische Bildung beschränkt sich jedoch nicht nur auf problemlösendes Tun, sondern umfasst auch das analysierende technische Denken als gedankliches Durchdringen technischer Prinzipien, Funktionsweisen und Prozesse wie auch das Bewerten und Kommunizieren von Technik." (63). Unabhängig von den jeweils zugrundeliegenden didaktischen Konzeptionen gilt, dass der Unterricht handlungsorientiert konzipiert und umgesetzt werden sollte (vgl. Dölle, Lambert \& Zolg, 2013). Im Perspektivrahmen werden für die technische Perspektive zudem sechs "grundlegende Inhaltsfelder von Technik“ benannt. Diese umfassen „Arbeit und Produktion, Bauen und Wohnen, Transport und Verkehr, Ver- und Entsorgung, Haushalt und Freizeit, Information und Kommunikation" $(2013,63)$. Die unterrichtliche Auseinandersetzung mit Technik wird somit in ein breites Spektrum gesetzt, das gesellschaftliche Bezüge integriert. 


\section{Forschungsstand zur Ausstattung zum technischen Lernen an (Grund)Schulen}

Technische und auch informatische Lernprozesse können grundsätzlich auch ohne eine besondere Ausstattung oder Informatiksysteme stattfinden (vgl. Bergner u. a. 2018, 245). So zeigen z. B. Bergner et al. (2018) mittels einiger Beispiele auf, wie informatische Bildungsprozesse „unplugged“ mit einfachen Materialien, wie Papier und Stiften, initiiert werden können. Dennoch bieten Werkzeuge, ob handwerklich oder digital, zusätzliches Potential sowohl im Bereich der Denk-, Arbeits- und Handlungsweisen (vgl. GDSU 2013), der Motivation und der Lernerfahrungen (vgl. Bergner u. a. 2018, 245) als auch in inhaltlichen Bereichen der Technischen Bildung (vgl. Dölle u. a., 2013). Technische Lernprozesse können nach den oben ausgeführten Darlegungen unterschiedliche Themen bzw. gesellschaftliche Bezüge aufweisen, wobei auch die Digitalisierung zu integrieren ist. Daher sind inzwischen nicht mehr nur handwerkliche Werkzeuge von Bedeutung für das technische Lernen in Schulen, sondern auch digitale. Hinsichtlich der Ausstattung von Schulen zur Technischen Bildung stellt sich daher die Frage, in welchem Umfang was vorhanden ist, um technische Bildung de facto zu ermöglichen.

Neuere Studien, die thematisch die technischen Ausstattungsbedingungen an Schulen untersuchen, konzentrieren sich vermehrt auf digitale Medien (vgl. Bos u. a. 2013, Cornelsen Trendstudie 2015, Deutsche Telekom-Stiftung 2017). Die Studien belegen, dass mit 42,5\% nicht einmal die Hälfte der befragten Lehrerinnen und Lehrer in Niedersachsen die Ausstattungsbedingungen im Bereich der Informatiksysteme als ausreichend bewerten (Deutsche Telekom-Stiftung 2017, 10). Auch die eigene Zufriedenheit mit der Medienausstattung an ihrer Grundschule liegt bei Grundschullehrkräften lediglich im mittleren Bereich (genauer bei 3,6 auf einer sechsstufigen Likert-Skala, Cornelsen Trendstudie 2015, 5). Bei dieser Studie bleibt allerdings offen, welche Verbesserungen die befragten Lehrkräfte als sinnvoll bzw. lernförderlich erachten würden.

Für Grundschulen zeigten Breiter et al. (2013) in einer Schulbefragung auf, dass das Verhältnis von Schülerinnen und Schülern pro Computer in Deutschland im Jahr 2011 im Durchschnitt bei neun zu eins lag (171). Die Erhebung gibt jedoch in Bezug auf das Alter, die Ausstattung und die Funktionsfähigkeit der Geräte keine Antworten, da hierzu keine Teilfragen erhoben wurden.

Auf Grundlage der bisherigen Entwicklung, die ein stetiges Ansteigen der Anzahl von Informatiksystemen an Schulen aufzeigt, ist zu vermuten, dass sich dieses Verhältnis in den letzten Jahren weiter veränderte. Insofern ist es überraschend, dass in Deutschland im internationalen Vergleich die Nutzung digitaler Informatiksysteme nicht zufriedenstellend ist. Die ICIL-Studie („International Computer and Information Literacy Study") zeigt, dass in keinem anderen Teilnehmerland 
Computer seltener im Unterricht eingesetzt werden als in Deutschland (Bos u. a. 2014). Auch im Folgejahr lag Deutschland erneut in der OECD-Studie „Students, Computers and Learning" (OECD 2015) in der Nutzung von Computern im Mathematikunterricht auf Platz 27 und damit weit unter dem OECD Durchschnitt. ${ }^{1}$ Die Studie bezieht sich auf die Sekundarstufe. Es kann nur vermutet werden, dass eine Erhebung in Grundschulen ähnliche Ergebnisse erbringen würde. Denn einerseits werden digitale Medien als relevant von Lehrenden angesehen, der Einsatz im Unterricht scheint jedoch nicht mit dieser Einschätzung einherzugehen: So plädieren über ein Drittel der Grundschullehrkräfte (35\%) für eine wichtige Rolle der digitalen Medien in der Grundschule, nur zehn Prozent sehen diese jedoch realisiert (IfD Allensbach 2013, 4). Dies deckt sich mit der Sichtweise der Grundschüler*innen, von denen zwei Drittel in dieser Befragung angaben, dass digitale Medien in ihrem Unterricht eine weniger große (51\%) oder sogar kaum bis gar keine Rolle (15\%) spielen (ebd.).

Es stellt sich die Frage, woraus diese Inkongruenz resultiert. Neben lehrerbezogenen Variablen wie Einstellung zu technischen Themen, Sozialisation und Ausund Fortbildungshintergründen, sind auch die Ausstattungsbedingungen als ein Einflussfaktor zu vermuten.

Schon die bereits in den 1990er Jahren durchgeführte quantitative Studie zum „Technischen Lernen im Sachunterricht" erkennt die Ausstattungsbedingungen von Schulen als eine relevante Variable für die Realisierung technikbezogenen Sachunterrichts (Möller u. a. 1996, 2). Es zeigt sich jedoch bereits seit der ersten Erhebung technischer Ausstattungsbedingungen an Grundschulen in den 1990er Jahren, dass Lehrerinnen und Lehrer diese als mangelhaft wahrnehmen (Möller u. a. 1996, 43).

Wichtig ist hierbei, dass keine Fehlschlüsse interpretiert werden: Vorhandene Medien oder Werkzeuge lassen keine Rückschlüsse auf deren tatsächlichen Nutzung zu. Die didaktischen Möglichkeiten sind nicht mit einer unterrichtlichen Umsetzung gleichzusetzen. Letztendlich hängt die Qualität unterrichtlicher Prozesse von einer Vielzahl an Variablen ab, die in einem komplexen Wirkzusammenhang stehen (vgl. z. B. Helmke, 2015). Die Ausstattungsbedingungen zeigen sich demnach als eine Variable in der Diskussion um Unterrichtsqualität (vgl. ebd.). Denn nur, wenn Werkzeuge, Materialien und Medien verfügbar sind, können sie auch eingesetzt werden (vgl. Breiter u. a. 2013, 169).

Zusammenfassend können Einseitigkeiten in Bezug auf die empirischen Erhebungen zur technischen Ausstattungssituation an Schulen festgestellt werden. Die Schwerpunkte liegen in den Sekundarschulen und hierbei zudem bei der Erhe-

1 Erhoben wurde die Prozentzahl der Schülerinnen und Schüler, die angaben, dass sie während des letzten Monats vor Durchführung der PISA-Studie einen Computer im Mathematikunterricht genutzt haben (OECD, 2015, 57) 
bung von digitalen Medien. Die Ist-Situation bezüglich der technischen digitalen Ausstattung an Grundschulen wurde bislang lediglich über die Einschätzung von Lehrerinnen und Lehrer erhoben. Es stellt sich auch die Frage, vor welchem Hintergrund die befragten Lehrerinnen und Lehrer ihre Beurteilung vorgenommen haben. So erarbeiteten Breiter u. a. (2017) beispielsweise Ausstattungsszenarien für allgemeinbildende Schulen, in denen sie eine idealtypische, lernförderliche IT-Infrastruktur aufzeigen, die unabhängig von der pädagogisch-didaktischen Ausrichtung angenommen wird (Breiter u. a. 2017,2). Inwieweit sich die Idealausstattung von Grundschulen mit der an den Schulen vorhandenen Ausstattung deckt, kann anhand der vorliegenden Studien nicht beurteilt werden. So wurde in der Studie des IfD Allensbach (2013) zwar erhoben, ob Computerräume vorhanden sind, allerdings wurde dies lediglich durch die Angabe von Lehrer*innen ermittelt ( $82 \%$ der Lehrer*innen gaben an, dass an ihrer Schule ein Computerraum vorhanden sei, ob hier jedoch als Klassenverband gelernt werden kann, bleibt offen). Vor dem Hintergrund von Lehrer*innenaus- und -fortbildungen, fachdidaktisch methodischen Perspektiven und der bildungspolitisch geforderten verstärkten Integration technisch medialen Lernens in den Unterricht (KMK 2016), ist aber genau das ein entscheidender Aspekt. Die im Folgenden vorgestellte eigene empirische Studie widmet sich deshalb auch der Frage, inwiefern es, unabhängig von der tatsächlichen Nutzung im Unterricht, Möglichkeiten für Schulklassen gibt, im Klassenraum oder in spezifischen Unterrichtsräumen technische Lernprozesse zu erfahren.

\section{Design der Pilotstudie: Technische Ausstattung an Praxisgrundschulen}

In der vorgestellten Studie wird die Frage nach den Ausstattungssituationen an Praxisgrundschulen hinsichtlich des technischen Lernens gestellt. Da technischer Sachunterricht handlungsorientiert angelegt sein sollte (vgl. Mammes \& Tuncsoy 2013), ist die Erhebung der Ausstattungssituationen von Grundschulen grundlegend bedeutsam.

Die hier vertretene konzeptionelle Sicht auf technische Bildung schließt für das technische Lernen im Sachunterricht u. a. auch die sozialwissenschaftliche Perspektive mit (vgl. z. B. Ropohl, 2009, 39). Denn Technik ist stets von Menschen konstruiert und insofern auch in Bezug auf soziale und ethische Potentiale und Gefahren, auf ästhetische Aspekte und zudem auf eine Vielzahl weiterer Bereiche zu beleuchten. Hierfür können Filme, Schaubilder, Sachtexte etc. sinnhaft und lernförderlich sein. Diese können u. a. auch in Kindersachbüchern oder Schulbüchern enthalten sein. In der vorgestellten Studie wird der Fokus allerdings vor 
allem auf die handwerkliche und digitale Ausstattungssituation gelegt. Somit wird nicht die gesamte mögliche Ausstattung für das technische Lernen erhoben. Die Erfassung erfolgt durch Erhebungsbögen, in denen relevante Werkzeuge, Materialien und Medien in bereits im Vorfeld rekonstruierten theoriegeleiteten Kategorien erhoben werden. Hierfür wurde die übergeordnete Fragestellung in zwei Fragenkomplexe unterteilt, die jeweils die Grundlage für die Entwicklung des Erhebungsbogens war.

\subsection{Werkräume und ihre Ausstattung zum technischen Lernen}

Werkräume sind keine Voraussetzung für technisch handwerkliches Lernen, allerdings bieten sie eine spezifischere Ausstattung als Klassenräume. Sie zeichnen sich durch Werkbänke und Werkzeuge aus. Zwar sind sie für die Arbeit mit Werkzeugen nicht entscheidend, so können die Tische auch mit Abdeckplatten geschützt werden (Möller, 2015). Der sachgerechte Umgang mit Werkzeugen wird jedoch vereinfacht. In Bezug auf das vor Ort vorhandene Werkzeug, das Grundschüler*innen eigene Erfahrungen in technisch handwerklichen ausgerichteten Lernsituationen bietet, stellt sich zunächst die Frage, welche Grundausstattung für Grundschulen sinnvoll wäre. Möller (2015) empfiehlt in ihrer Praxishandreichung folgende Werkzeuge für die Arbeit mit Holz: Fein- und Laubsäge, Feile, Schleifpapier und Raspel, Nagelbohrer („Handbohrer“), Schraubendreher, Kneifzange, Hammer und Schraubzwinge.

Der erste Fragenkomplex enthält Fragen zu den technischen handwerklichen Ausstattungsbedingungen. Hierfür wurden folgende Forschungsfragen formuliert:

1. Gibt es an den untersuchten Grundschulen Werkräume?

2. Wenn ja, wie sind diese Räume mit Werkzeugen ausgestattet?

3. Wenn nein, inwiefern wird dann Werkzeug für die Nutzung durch Schüler*innen anderweitig aufbewahrt?

In der Studie wurde deshalb die Ausstattung mit Werkbänken und den o.g. Werkzeugen in Werkräumen erhoben, ebenso auch Werkzeuge, die in anderen Räumen oder in Klassenräumen aufbewahrt werden. Somit konnte eine verkürzte bzw. eingeschränkte Darstellung der vorhandenen Werkzeuge vermieden werden.

\subsection{Computer- und Klassenräume und ihre Ausstattung zum technischen Lernen}

Es ist ein Unterschied, ob in einem Klassenraum einige Computer, Notebooks oder Tablets zur Einzelnutzung zur Verfügung stehen oder ob eine ganze Klasse in einem eigens dafür konzipierten Raum lernen kann. Insofern wurde in einem ersten Schritt zunächst erhoben, ob es in den Schulen Computerräume gibt und wie diese ausgestattet sind. Um zu überprüfen, ob an diesen Computern gearbei- 
tet werden kann, wurden einige Bedingungen zudem überprüft. So bestimmt das Alter eines Computers zum Teil mit, welches Betriebssystem zur Wahl steht und welche Programme und Apps problemlos auf dem Gerät genutzt werden können. Ein Internetzugang erweitert das Spektrum der Nutzungsmöglichkeiten z. B. um Recherchemöglichkeiten. Drucker können genutzt werden, um Ergebnisse festzuhalten und in den Klassenräumen damit weiterzuarbeiten, wenn dort keine Computer o.ä. zur Verfügung stehen. Insofern wurden das Alter der Computer, das Betriebssystem und die Version, der Internetzugang und die Anzahl der vorhandenen Drucker erhoben. Zudem gilt, dass Computer, Notebooks und andere digitale Medien regelmäßig aktualisiert werden werden müssen, es gibt beispielsweise Updates für bestimmte Programme oder Apps. Deshalb wurde ebenfalls erhoben, ob es an den jeweiligen Schulen Medienbeauftragte gibt. Damit kann selbstverständlich keine Aussage getroffen werden, ob die vorhandene Software regelmäßig in den letzten Jahren aktualisiert wurde, aber die Möglichkeit wird damit ausgelotet. Da die mediale Ausstattung sich nicht nur auf Computer beschränkt, wurde zudem die weitere Ausstattung erhoben.

Um die Frage nach den medialen Ausstattungsbedingungen zu beantworten, wurden folgende Forschungsfragen formuliert:

1. Gibt es an den Praxisgrundschulen spezifische Computerräume, in denen die Schüler*innen als Klasse lernen können?

2. Wie sind die Klassenräume medial ausgestattet?

3. Gibt es Medienbeauftragte/-verantwortliche für die Wartung der Geräte?

\subsection{Durchführung der quantitativen Erhebung}

Die Analyse der Ausstattung anhand eines theoriegeleiteten Erhebungsbogens hat sich während der Durchführung als effizient herausgestellt. Da die Fragestellungen nicht die Einschätzung der Lehrerinnen und Lehrer umfassen, sondern den Ist-Zustand, wurde die Ausstattung vor Ort an den Schulen mittels eines kategoriengeleiteten Erhebungsbogens erfasst. Der kategoriengeleitete Erhebungsbogen ist so konstruiert, dass z. B. Gegenstände, die nicht in die vorliegenden Kategorien passen, aber relevant für die Fragestellung sind, ebenfalls erhoben werden können. Es wurde eine Prä-Pilotierung durchgeführt, in der für jede Oberkategorie auch eine offene Kategorie für zusätzliche Materialien und Anmerkungen aufgenommen wurde. Die offenen Kategorien der Prä-Pilotierung wurden bereits zur Ausschärfung des nun vorliegenden Instruments genutzt. Um die korrekte Durchführung der Erhebung zu gewährleisten, wurden die Durchführenden entsprechend geschult. Zudem wurden drei der Schulen doppelt erhoben, um die Interrater-Reliabilität sicherzustellen. Auch bei einer niedrig-inferenten Erhebung, wie sie in diesem Fall vorliegt, ist das Kappa von >.9 mehr als zufriedenstellend. 


\subsection{Begründung und Darstellung der Stichprobe}

Die im Folgenden vorgestellte Pilotstudie wurde an zwölf Praxisschulen im Raum Osnabrück durchgeführt. Die Fokussierung auf Praxisschulen wurde bewusst gewählt. Hintergrund ist, dass alle Studierenden für das Lehramt an Grund-, Hauptoder Realschulen seit dem WS 2014/15 im ersten Jahr ihres Masterstudiengangs ein Langzeitpraktikum (den Praxisblock) mit einer Dauer von 18 Unterrichtswochen an einer allgemeinbildenden Schule absolvieren. Die Neustrukturierung der Lehramtsmasterstudiengänge soll ermöglichen, dass Studierende im Praxisblock vermehrt Unterrichtssequenzen durchführen und dabei von Mentor*innen ihrer Praktikumsschule betreut werden. Ziel ist zudem, dass die Studierenden an ihrer Praktikumsschule erste Einblicke in die Strukturen bzw. das Berufsfeld von Lehrenden bekommen (Schulleben, Gremienarbeit, Ganztag etc.). Nach diesem Konzept sollen somit ausgewählte Schulen zum Lernort der Studierenden werden. Somit soll eine Praxis- und Berufsfeldorientierung im Masterstudium gewährleistet werden. Bedeutsam sind aber hinsichtlich der Qualität der Lehramtsausbildung nicht nur die beteiligten Akteure. Auch die Ausstattung der einzelnen Ausbildungsschulen gilt es kritisch zu betrachten. Denn durch ihre räumliche und materielle Ausstattung werden den Studierenden unterschiedliche Sichtweisen und Möglichkeiten eröffnet und Schulen als mehr oder weniger moderne Lernräume im Studium erfahren.

In der Pilotstudie wurden insgesamt 119 Klassenräume 2 sowie 89 Fach-, Material- und Differenzierungsräume untersucht. In der Tabelle 1 wird ein Überblick über die erfassten Schulen gegeben, wobei der Modus, in diesem Fall das häufigste Vorkommen, für acht der zwölf Schulen zutraf. An der kleinsten untersuchten Schule sind fünf Lehrerinnen beschäftigt und die insgesamt 76 Schülerinnen und Schüler verteilen sich auf vier Klassen. Dahingegen unterrichten an der größten untersuchten Schule 32 Lehrerinnen und Lehrer in insgesamt 20 Klassen, die 615 Schülerinnen und Schülern umfassen.

Tab. 1: untersuchte Grundschulen $(\mathrm{n}=12)$

\begin{tabular}{|l|r|r|r|r|}
\hline & Schule min. & $\begin{array}{l}\text { Schule } \\
\text { max. }\end{array}$ & $\begin{array}{l}\text { häufigstes } \\
\text { Vorkommen } \\
\text { (Modus) }\end{array}$ & Mittelwert \\
\hline $\begin{array}{l}\text { Anzahl der } \\
\text { Schüler*innen }\end{array}$ & 76 & 615 & $170-210$ & 221 \\
\hline $\begin{array}{l}\text { Anzahl der } \\
\text { Lehrer*innen }\end{array}$ & 5 & 32 & $12-14$ & 17 \\
\hline $\begin{array}{l}\text { Anzahl der } \\
\text { Klassen }\end{array}$ & 4 & 20 & $10-12$ & 10 \\
\hline
\end{tabular}

2 Die zwölf Schulen verfügen insgesamt über 124 Klassenräume, wobei fünf der Räume in dieser Studie nicht erfasst werden konnten. 


\section{Darstellung der Ergebnisse}

\subsection{Werkräume in Praxisschulen und ihre Ausstattung}

Von den untersuchten 12 Schulen verfügen 11 über einen Raum, der nach Auskunft der Schulen als Werkraum genutzt werden kann. Allerdings konnte durch die konkrete Erhebung vor Ort erfasst werden, dass es sich in einem Fall nur bedingt um einen Werkraum handelt, da zum einen keine Werkbänke zur Verfügung stehen, um zum anderen lediglich unterschiedliche Werkzeuge in diesem Raum gelagert werden. In den 11 erfassten Werkräumen sind Werkbänke in einer Anzahl von vier bis neun vorhanden (vgl. Tabelle 2).

Tab. 2: Anzahl der Werkbänke in den Werkräumen

\begin{tabular}{|l|r|r|r|}
\hline $\begin{array}{l}\text { Anzahl der } \\
\text { Werkbänke }\end{array}$ & Häufigkeit & Klassenzahl & \multicolumn{2}{|l|}{ Schülerzahl } \\
\hline Keine & 1 & 10 & 203 \\
\hline Zwei & 1 & 14 & 305 \\
\hline Vier & 3 & $9,10,20$ & $186,206,615$ \\
\hline Sechs & 4 & $8-11$ & $104,198-203$ \\
\hline Acht & 1 & 8 & 172 \\
\hline Neun & 1 & 12 & 208 \\
\hline
\end{tabular}

In Bezug auf die Zugänglichkeit der Werkräume konnte erfasst werden, dass alle Lehrer*innen gleichermaßen, unabhängig von ihrer Fächerkombination, diese Räume nutzen können. In einem weiteren Schritt wurden erhoben, welche Werkzeuge in den Werkräumen vorhanden sind. Dabei wurde auch untersucht, inwieweit Klassensätze bzw. einzelne Werkzeuge verfügbar sind. In keiner der untersuchten Schulen liegen Klassensätze aller von Möller (2015) empfohlenen Werkzeuge im Werkraum vor. Hammer und Nagelbohrer stehen in sieben bzw. vier Schulen als Klassensatz zur Verfügung. Nur an einer Schule ist beides nicht vorhanden, ansonsten kann jeweils auf einzelne Hammer und Nagelbohrer zurückgegriffen werden. Zangen und Sägen liegen an jeweils zehn Schulen als Klassensatz vor, an zwei Schulen jedoch überhaupt nicht. Schraubendreher und Schraubzwingen fehlen an vier der zwölf Schulen, sind sonst aber vereinzelt oder als Klassensätze vorhanden. Um ausschließen zu können, dass sich weiteres Werkzeug in einzelnen Klassenräumen und weiteren Räumen (wie Differenzierungsräumen) befindet, wurden auch in diesen das Vorhandensein von Werkzeugen erhoben. So konnte beispielsweise über diese konsequente Vor-Ort-Erhebung erfasst werden, dass in der Schule, die keinen Werkraum besitzt, trotzdem Hammer, Sägen, Zangen, Schraubendreher, Feilen und Schraubzwingen als halbe 
Klassensätze vorliegen. Lediglich Nagelbohrer sind nur viermal vorhanden. Die Werkzeuge befinden sich im Lehrmittelraum und sind für alle Lehrerinnen der Schule zugänglich. Ein Arbeiten mit Werkzeugen ist an dieser Schule daher auch gewährleistet.

Lediglich in einer Schule werden einzelne Werkzeuge in einem Differenzierungsraum aufbewahrt. Ansonsten sind weder in den Klassenräumen noch in sonstigen Räumen weitere Werkzeuge vorhanden.

\subsection{Zusammenfassung und Diskussion}

Wie bereits dargestellt, verfügen alle bis auf eine der untersuchten Praxisschulen über einen Werkraum. Diese sind allerdings unterschiedlich ausgestattet, insbesondere das Verhältnis zur Anzahl der Klassen bzw. der Schüler*innen offenbart eine große Differenz. Die Ausstattungssituation zeigt zudem, dass es in den Schulen teilweise nur bedingt möglich ist, mit allen Schüler*innen einer Klasse technische Problemlöseprozesse organisatorisch umzusetzen. Denn nicht in allen Werkräumen können die Schüler*innen gleichzeitig an Werkbänken arbeiten. Da nur in zwei Fällen Werkzeug auch außerhalb des Werkraums aufbewahrt wird, scheinen alle handwerklichen Lernsituationen eher nicht in den Klassenräumen vorgesehen zu sein. Ob die Arbeit in den Werkräumen allen Lernenden durch flexible Organisationsformen ermöglicht wird, um allen Schüler*innen gleichermaßen Möglichkeiten zur Arbeit mit Werkzeugen zu geben, gilt es in einem weiteren Schritt zu im Detail zu hinterfragen.

\subsection{Computerräume in Praxisschulen und ihre mediale Ausstattung}

Während elf untersuchten Praxisschulen einen Werkraum besitzen, findet sich nur an sechs der zwölf untersuchten Schulen ein Computerraum. Die Anzahl der Computer variiert zwischen zwölf und 29 Einzelcomputern, so dass die meisten Schüler*innen zu zweit oder auch allein an einem Computer arbeiten können. Die Computer sind stationär installiert. Notebooks und Tablets sind in allen Computerräumen nicht vorhanden. Wie die Tabelle 3 zeigt, sind nur zwei der Computerräume mit unter drei Jahre alten Computern ausgestattet. Alle anderen Computer sind älter, in einem Computerraum sogar älter als sechs Jahre. Dies bedingt auch die jeweiligen auf den Rechnern befindlichen Betriebssysteme. In allen Schulen wird mit Windows gearbeitet. Allerdings laufen nur die Computer zweier Schulen mit dem zurzeit aktuellen „Windows 10“, alle anderen Computer sind mit „Windows 7“ oder sogar einem älteren Betriebssystem ausgestattet. Das Betriebssystem „Windows 7“ war von 2009 bis 2011 die Hauptversion der Microsoft Betriebssysteme und wird heute nur eingeschränkt unterstützt. 
Tab. 3: PCs in den Computerräumen

\begin{tabular}{|l|r|l|l|r|}
\hline $\begin{array}{l}\text { Schule } \\
\text { PC- } \\
\text { Raum }\end{array}$ & $\begin{array}{l}\text { Anzahl } \\
\text { der PCs }\end{array}$ & $\begin{array}{l}\text { Alter der } \\
\text { PCs }\end{array}$ & Betriebssystem & $\begin{array}{l}\text { Anzahl } \\
\text { der SuS }\end{array}$ \\
\hline A & 22 & $2012-13$ & Win7 oder älter & 198 \\
\hline B & 27 & $2016-17$ & Win10 & 615 \\
\hline C & 12 & $\begin{array}{l}\text { älter als } \\
2012\end{array}$ & Win7 oder älter & 206 \\
\hline D & 27 & $2016-17$ & Win7 & 305 \\
\hline E & 29 & $\begin{array}{l}2012 \text { und } \\
\text { älter }\end{array}$ & Win7 oder älter & 205 \\
\hline F & 27 & $2014-15$ & Win10 & 203 \\
\hline
\end{tabular}

Die Ausstattung mit Druckern ist nicht in jedem Computerraum gewährleistet. So gibt es in einem Computerraum einen Farbdrucker, in drei Räumen steht jeweils ein schwarz-weiß Drucker zur Verfügung, in zwei Räumen ist gar kein Drucker vorhanden. Zusätzlich zu den Computern sind in drei Computerräumen stationäre, interaktive Whiteboards mit Internetzugang vorhanden. Eines der Whiteboards wurde vor 2012 angeschafft, die anderen beiden sind aus den Jahren 2016/2017. Ebenfalls sind jeweils in drei der sechs Computerräumen ein Beamer installiert. Film- oder Diaprojektoren oder andere analoge Geräte sind in keinem der Räume vorhanden. Die sechs Computerräume sind für alle Lehrer*innen der jeweiligen Schule zugänglich. An acht der zwölf Schulen übernimmt eine der Lehrpersonen jeweils die Aufgabe der oder des Medienbeauftragten. Allerdings gibt es nur an vier der Schulen mit Computerräumen auch gleichzeitig eine/n Medienbeauftragte/n. An einer der Schulen mit dem aktuellen Betriebssystem gibt es beispielsweise keine Person, die für die Wartung der Computer zuständig ist oder Treiberprobleme beheben kann.

\subsection{Klassenräume und ihre mediale Ausstattung in Praxisschulen}

Da nicht alle Grundschulen, wie bereits ausgeführt, über einen Computerraum verfügen, gilt es auch zu überprüfen, welche mediale Ausstattung die Praxisgrundschulen insgesamt besitzen, d.h. vor allem in den einzelnen Klassenräumen. Die Untersuchung ergab, dass sich in 70 der 119 untersuchten Klassenräumen keine Computer befinden. In den 49 ausgestatteten Klassenräumen stehen ein bis drei Computer jeweils zur Verfügung (siehe Abbildung 1). In drei der 49 Klassenräumen befindet sich zusätzlich jeweils ein Notebook. Die inzwischen in privaten Haushalten sehr verbreiteten Tablets sind in keinem Klassenzimmer vorhanden. Die Daten zeigen einen Zusammenhang zwischen dem Vorhandensein eines 
Computerraumes und den Computern in den Klassenräumen. ${ }^{3}$ Von den 70 Klassenräumen, in denen keine Computer vorhanden sind, können 45 Klassen einen schuleigenen Computerraum nutzen. Das bedeutet aber auch umgekehrt, dass 25 Schulklassen, das entspricht über 20\% der Stichprobe, weder einen Zugriff im eigenen Klassenraum noch in einem spezifischen Raum auf einen Computer haben.

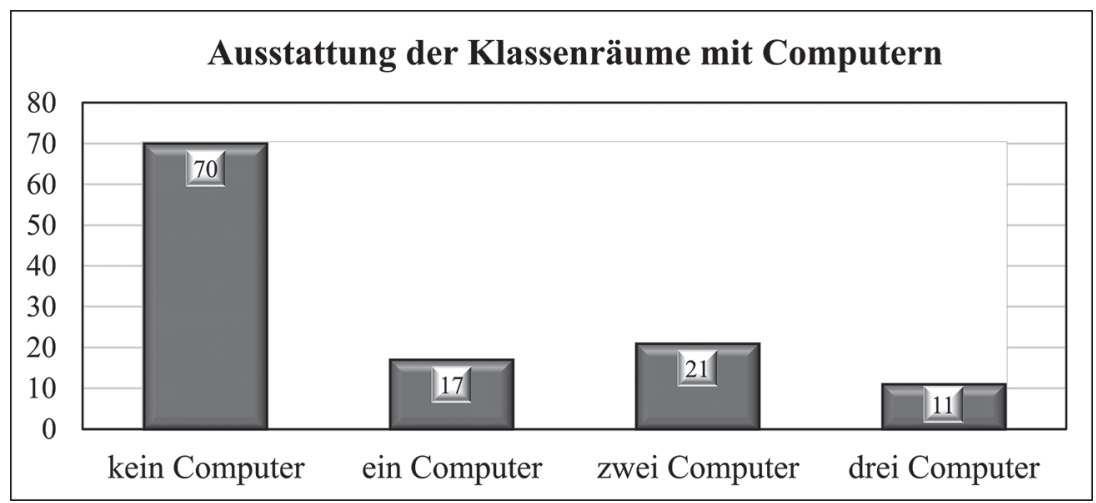

Abb. 1: Computer in den Klassenräumen $(n=119)$

Da technische und digitale Bildung den gesamten Grundschulbereich umfasst, also vom Anfangsunterricht bis in die höheren Klassenstufen, ist auch von Interesse, wie die Computer sich über die Klassenräume der einzelnen Jahrgangsstufen verteilen. Wie die Abbildung 2 zeigt, ist die Verteilung so, dass auch erste und zweite Klassen teilweise einen Zugang zu einem Computer bereits haben.

3 Die statistisch signifikante Korrelation $(r=.195, p=.034)$ ist aber aufgrund der geringen Stichprobe vorsichtig zu interpretieren. 


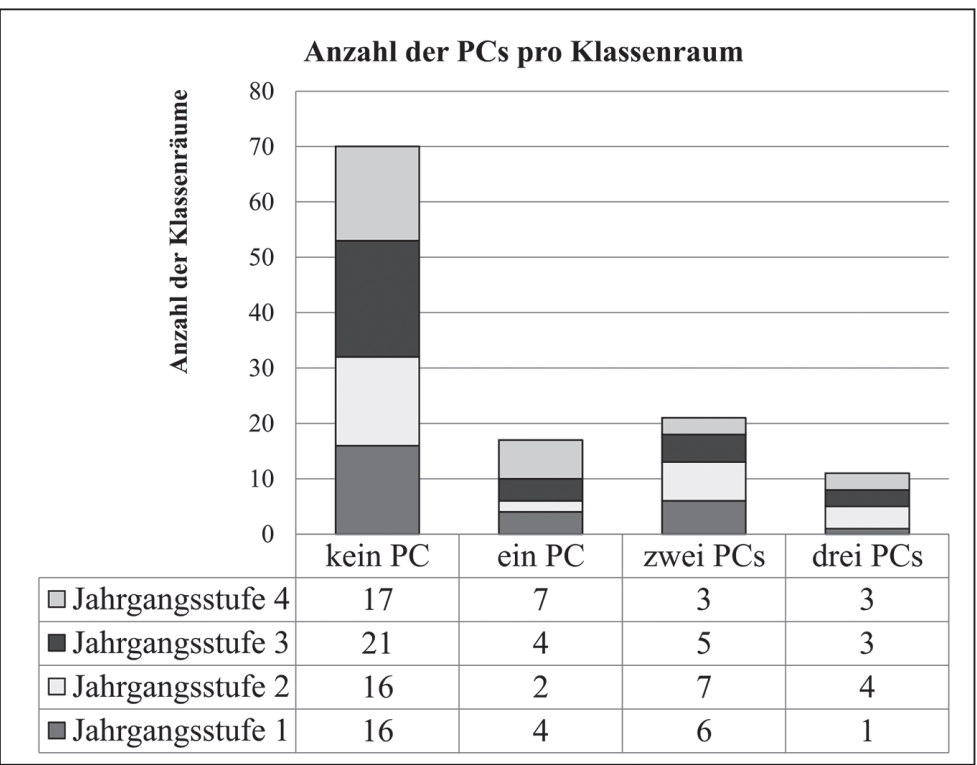

Abb. 2: Ausstattung der Klassenräume mit Computern pro Jahrgangsstufe

Auch bei den Geräten in den Klassenräumen wurde das Alter der Computer erhoben. Es zeigt sich, dass die meisten Computer aus dem Jahr 2012 oder älter sind (Abbildung 3). Das Diagramm stellt dar, wie alt die Computer in den jeweiligen Klassen sind.

\section{Alter der Computer}
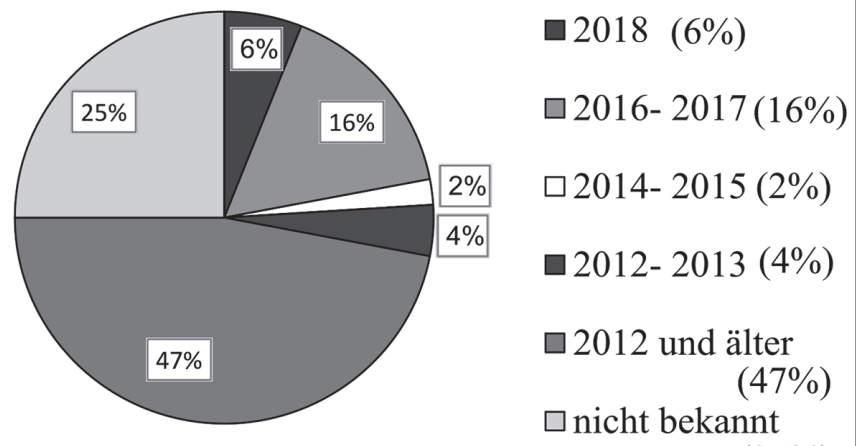

$(25 \%)$

Abb. 3: Alter der Computer in den Klassenräumen 
Auch in Bezug auf die Betriebssysteme gibt es keine Unterschiede zwischen den spezifischen Räumen und den Klassenräumen. Denn auch die Computer in den Klassen haben Windows basiert. Die jeweilige Version variiert zwischen Windows 8 und 10, in vier Klassen sind noch ältere Versionen (Windows 7 oder älter) installiert.

\subsection{Weitere mediale Ausstattung}

In den 49 Klassenräumen, die über Computer verfügen, steht auch ein Internetzugang zur Verfügung. In 15 Fällen ist das Internet für die Schülerinnen und Schüler nicht direkt zugänglich, da nur die Lehrerinnen und Lehrer über das nötige Passwort verfügen. In 34 Klassenräumen haben die Schülerinnen und Schüler dagegen freien Zugang zum Internet.

Zudem wurde die weitere digitale mediale Ausstattung erhoben. Überraschend ist, dass sich nur in drei Klassenräumen ein Drucker befindet. Über diese weitere mediale Ausstattung gibt die Tabelle 4 Auskunft: Interaktive Whiteboards sind in keinem der Klassenräume vorhanden, dafür gibt es in fast allen Klassen Kreidetafeln (in 114 von 119 Klassenräumen), in 38 Klassen gibt es einen Beamer.

Tab. 4: Weitere mediale Ausstattung ( $\mathrm{n}=119)$

\begin{tabular}{|l|r|r|r|}
\hline & $\begin{array}{l}\text { Anzahl der } \\
\text { Klassen }\end{array}$ & MW* & \multicolumn{1}{l|}{ SD* } \\
\hline Interaktives Whiteboard & 0 & 1 & 0,3 \\
\hline Fernseher & 1 & 0,1 & 0,4 \\
\hline Drucker & 3 & 0 & 0 \\
\hline Overheadprojektor & 17 & 0,3 & 0,5 \\
\hline $\begin{array}{l}\text { Dokumentenkamera } \\
(, \text { Elmo })\end{array}$ & 31 & 0 & 0,2 \\
\hline Beamer & 38 & 0 & 0 \\
\hline Kreidetafel & 114 & 1 & 0,4 \\
\hline Musikanlage & 115 & 0,3 & 0,4 \\
\hline
\end{tabular}

* Mittelwert (MW) und Standardabweichung (SD), die Werte sind gerundet

\subsection{Medienbeauftragte und Medienkonzept}

An acht der zwölf Schulen übernimmt eine der Lehrpersonen jeweils die Aufgabe der oder des Medienbeauftragten. Allerdings gibt es nur an vier der Schulen mit Computerräumen auch gleichzeitig eine/n Medienbeauftragte/n. Die vorliegenden Daten zeigen dabei keinen Zusammenhang zwischen der Aktualität der Geräte und dem Vorhandensein eines Medienkonzeptes und/oder eines Medienbeauftragten. An drei der zwölf Praxisschulen lag zum Zeitpunkt der Erhebung 
noch kein Medienkonzept vor. Die vorliegenden Daten zeigen dabei keinen $\mathrm{Zu}$ sammenhang zwischen der Aktualität der Geräte und dem Vorhandensein eines Medienkonzeptes und/oder von Medienbeauftragten.

\subsection{Zusammenfassung und Diskussion}

In über der Hälfte der Klassenräume der erhobenen Praxisgrundschulen befindet sich kein Computer (in 70 von 119 Klassenräumen). Für 45 dieser Klassen besteht allerdings die Möglichkeit in einem Computerraum zu arbeiten. Anzumerken ist aber, dass eine situative, adaptive Nutzung im regulären Unterricht damit nur bedingt möglich sein kann. Auch erfordert der Raumwechsel zudem auch das zeitliche und inhaltliche Unterbrechen der Lernprozesse. Es kann darüber hinaus nicht davon ausgegangen werden, dass alle vorhandenen Computer für alle Zwecke einsatzbereit sind. Zumal das Alter der Computer und ihre Betriebssysteme die Vermutung nahelegen, dass eine spontane Nutzung im Unterricht nur eingeschränkt möglich ist. Das Hochfahren und Aktualisieren der älteren Systeme kann zeitaufwändig sein, zumal nicht immer eine regelmäßige Wartung gewährleistet ist. Das gilt ebenso für die Computer in den Computerräumen. Da nicht alle Schulen Medienbeauftragte haben, die sich um die Wartung der Geräte kümmern, ist anzunehmen, dass sich daraus eine zusätzliche Nutzungshürde ergibt. Es fällt zudem auf, dass keine Drucker in den Klassenräumen vorhanden sind. Somit können z. B. keine Rechercheergebnisse zur weiteren Verwendung ausgedruckt werden. Das kann insbesondere für Lernprozesse in den Klassenräumen schwierig sein, da es nur wenige Computer gibt und die Nutzungszeit für die einzelnen Schüler*innen insofern eingeschränkt ist. Auch in den Computerräumen können Rechercheergebnisse, Schreibdateien o.ä. nicht immer ausgedruckt und somit weiterhin verwendet werden.

\section{Fazit}

Für die Praxisgrundschulen der Pilotstudie konnte gezeigt werden, dass diese nur bedingt ausgestattet sind, um handwerkliche Lernprozesse zu initiieren. Ebenso sind Mängel bei der digitalen Ausstattung vorhanden. Bedenkt man, dass diese Schulen im Rahmen der ersten Phase der Lehramtsausbildung einen besonderen Stellenwert besitzen, dann sollte mit Nachdruck eine rasche Verbesserung der Ausstattungssituation eingefordert werden. Die geringe Stichprobe, die bei dieser Pilotstudie berücksichtigt wurde, gilt es bei der generellen Beantwortung der Frage, ob die Ausstattung zur technischen Bildung mangelhaft ist oder nicht, zu berücksichtigen. Daher wird derzeit die Erhebung mit einer größeren Stichprobe erneut durchgeführt. 


\section{Literatur}

Bergner, N., Hubwieser, P., Köster, H., Magenheim, J., Müller, K., Romeike, R., Schroeder, U. \& Schulte, C. (2018): Frühe informatische Bildung - Ziele und Gelingensbedingungen für den Elementar- und Primarbereich. Opladen: Verlag Barbara Budrich.

Bergner, N., Köster, H., Magenheim, J., Müller, K., Romeike, R., Schroeder, U. \& Schulte, C. (2017): Zieldimensionen für frühe informatische Bildung im Kindergarten und in der Grundschule. Opladen: Verlag Barbara Budrich.

Bos, W., Eickelmann, B., Gerick, J., Goldhammer, F., Schaumburg, H., Schwippert, K., Senkbeil, M., Schulz-Zander, R. \& Wendt, H. (Hrsg.) (2014): ICILS 2013. Computer- und informationsbezogene Kompetenzen von Schülerinnen und Schülern in der 8. Jahrgangsstufe im internationalen Vergleich. Münster: Waxmann.

Breiter, A., Zeising, A. \& Stolpmann, B.E. (2017): IT-Ausstattung an Schulen: Kommunen brauchen Unterstützung für milliardenschwere Daueraufgabe. Bertelsmann Stiftung: Gütersloh. Online unter: https://www.bertelsmann-stiftung.de/fileadmin/files/BSt/Publikationen/GrauePublikationen/ IB_Impulspapier_IT_Ausstattung_an_Schulen_2017_11_03.pdf (Abrufdatum: 12.07.2020).

Breiter, A., Aufenanger, S., Averbeck, I., Welling, S. \& Wedjelek, M. (2013): Medienintegration in Grundschulen. Untersuchung zur Förderung von Medienkompetenz und der unterrichtlichen Mediennutzung in Grundschulen sowie ihrer Rahmenbedingungen in Nordrhein-Westfalen. Berlin: Vista Verlag.

Cornelsen-Schulbuchverlage (Hrsg.) (2015): Cornelsen Trendstudie Medienausstattung und -nutzung an allgemeinbildenden Schulen in Deutschland. Online unter: https://www.cornelsen.de/ $\mathrm{fm} / 1272 /$ Cornelsen-Trendstudie\%20Medienausstattung\%20an\%20Schulen\%202014.pdf (Abrufdatum: 27.01.2019)

Deutsche Telekom-Stiftung (Hrsg.) (2017): Schule digital: Der Länderindikator 2017. Online unter: https://www.telekom-stiftung.de/sites/default/files/files/media/publications/Schule_Digital_2017 _Web.pdf (Abrufdatum: 12.07.2020)

Dölle, S., Lambert, A. \& Zolg, M. (2013): Bauen und Wohnen - Ein Thema des Sachunterrichts. In: I. Mammes, (Hrsg.): Technisches Lernen im Sachunterricht. Nationale und internationale Perspektiven. Baltmannsweiler: Schneider Verlag Hohengehren, 104-126.

Gesellschaft für Didaktik des Sachunterrichts (Hrsg.) (2013): Perspektivrahmen Sachunterricht. Bad Heilbrunn: Klinkhardt Verlag.

Helmke, A. (2015): Unterrichtsqualität und Lehrerprofessionalität. Diagnose, Evaluation und Verbesserung des Unterrichts. 6. überarbeitete Auflage. Seelze: Klett-Kallmeyer.

Institut für Demoskopie Allensbach \& Deutsche Telekom-Stiftung (Hrsg.) (2013): Digitale Medien im Unterricht. Möglichkeiten und Grenzen. Online unter https:/www.ifd-allensbach.de/uploads/ tx_studies/Digitale_Medien_2013.pdf (Abrufdatum: 27.01.2019)

KMK - Kultusministerkonferenz (2012/2016): Medienbildung in der Schule. Ständige Konferenz der Kultusminister der Länder in der Bundesrepublik Deutschland.

Mammes, I. \& Tuncsoy, M. (2013): Technische Bildung in der Grundschule. In: Mammes, I. (Hrsg.): Technisches Lernen im Sachunterricht. Nationale und internationale Perspektiven. Schneider Verlag Hohengehren: Baltmannsweiler, 8-21.

Möller, K. (Hrsg.) (2015): Holz erleben - Technik verstehen. Praktische Unterrichtsideen und Materialien für die Grundschule. Seelze: Friedrich Verlag.

Möller, K. (2014): Technisches Lernen fördern. Grundschulmagazin, 82, 31-35.

Möller, K., Tenberge, C. \& Ziemann, U. (1996): Technische Bildung im Sachunterricht. Eine quantitative Studie zur Ist-Situation an nordrhein-westfälischen Grundschulen. Selbstverlag: Münster.

OECD (2015): Students, Computers and Learning: Making the Connection, PISA, OECD Publishing. http://dx.doi.org/10.1787/9789264239555-en (Abrufdatum: 12.07.2020)

Ropohl, G. (2009): Allgemeine Technologie - Eine Systemtheorie der Technik. 3., überarbeitete Auflage. Universitätsverlag: Karlsruhe. 